\title{
Differentially regulated ADAMTS1, 8, and 18 in gastric adenocarcinoma
}

\author{
Kilic $\mathrm{MO}^{1}$, Aynekin $\mathrm{B}^{2}$, Kara $\mathrm{A}^{3}$, Icen $\mathrm{D}^{4}$, Demircan $\mathrm{K}^{5}$ \\ Department of General Surgery, Numune Training and Research Hospital, Ankara, Turkey. \\ murat05ozgur@hotmail.com
}

\section{ABSTRACT}

OBJECTIVE: The aim is to investigate the expression status of ADAMTS1,8, and 18 proteases in gastric cancer (GC) and lymphatic metastasis.

BACKGROUND: A disintegrin and metalloprotease with thrombospondin motifs (ADAMTS) is a new protease family, and has important biological functions such as hemostasis, extracellular matrix remodeling and regulation of angiogenesis.

METHODS: The immunostaining status of ADAMTS1,8, and 18 were investigated in formalin-fixed paraffinembedded samples of 25 patients who underwent curative resection for GC.

RESULTS: The immune reactivity scores (IRS) of ADAMTS1, 8, and 18 were significantly higher in the cancerous gastric tissue in comparison to non-cancerous gastric tissue $(p<0.001)$. In addition, IRS scores of these three ADAMTS proteases were higher in the metastatic lymph nodes compared with healthy lymph nodes $(p<$ 0.001 ). The expression status of the three ADAMTSs in cancerous gastric tissue was correlated with stage of tumor. Additionally, ADAMTS1 expression and ADAMTS8 expression in cancerous gastric tissue were found to correlate with grade and tumor size, respectively.

CONCLUSION: This study showed that ADAMTS1, 8, and 18 are highly expressed in GC and its nodal metastases, suggesting important roles of these proteases in carcinogenesis and lymphatic metastasis. The findings from the present study indicate that these proteases may be promising candidates for novel and alternative treatments in GC (Tab. 3, Fig. 3, Ref. 27). Text in PDF www.elis.sk.

KEY WORDS: ADAMTS1, ADAMTS8, ADAMTS18, immunohistochemistry, gastric cancer.

\section{Introduction}

Gastric cancer (GC) is the fourth most common type of cancer and the second most common cause of death among all cancers worldwide, despite a significiant decline in its incidence (1). Both genetic and environmental factors such as Helicobacter pylori infection, bad eating habits, and smoking play role in the etiogenesis of GC. Adenocarcinoma is the most common type of GC and represents approximately $95 \%$ of all cases. The majority of the patients are asymptomatic or have non-specific symptoms until the advanced stage of the disease. Therefore, only $25 \%$ of patients have localized disease at the time of diagnosis while one third of cases have distant metastases (2). Surgery is the primary treatment modality, but the overall 5-year survival rate is approximately

${ }^{1}$ Department of General Surgery, Numune Training and Research Hospital, Ankara, Turkey, ${ }^{2}$ Department of Medical Genetics, School of Medicine, Turgut Ozal University, Ankara, Turkey, ${ }^{3}$ Department of Histology and Embryology, Faculty of Veterinary Medicine, Atatürk University Erzurum, Turkey, ${ }^{4}$ Department of Statistics, Faculty of Science, Hacettepe University, Ankara, Turkey, and ${ }^{5}$ Department of Medical Biology, School of Medicine, Turgut Ozal University, Ankara, Turkey

Address for correspondence: M.O. Kilic, MD, Department of General Surgery, Numune Training and Research Hospital, Talatpaşa bulvari No. 44, Altindag, Ankara, Turkey.

Phone: +90.5053573122 , Fax: +90.3123061443
20-25 \% (3). Radiotherapy and chemotherapy have also limited effectiveness in prognosis. Therefore, novel diagnostic biomarkers and therapeutic targets are of great importance for earlier diagnosis and better prognosis of GC. In this context, most researches have focused on the genetic or molecular basis of GC in recent years. A disintegrin and metalloprotease with thrombospondin motifs (ADAMTS) is a family of 19 secreted membrane-anchored proteases, and is involved in various important biological processes such as hemostasis, extracellular matrix (ECM) remodeling and the regulation of angiogenesis associated with carcinogenesis and metastasis (4). Among those, ADAMTS1, 8, and 18 are classified as aggrecanases. Additionally, ADAMTS1 and 8 have anti-angiogenic property while ADAMTS18 is known as a tumor suppressor gene.

In this study, the expression status of ADAMTS1,8, and 18 in gastric adenocarcinoma and the association of these proteases with tumor characteristics were investigated using immunohistochemistry.

\section{Materials and methods}

\section{Patients and study design}

Twenty five patients who underwent surgery for gastric adenocarcinoma were included in this study. Total or subtotal gastrectomy with D1/D2 dissection was performed in all cases. Of 25 patients, 5 had stage 2 tumor and 20 had stage 3 tumor, according to the current 
Tab. 1. Immunoreactivity scoring system (IRS)* (6).

\begin{tabular}{|c|c|c|c|}
\hline Positive cells (\%) & Staining intensity & IRS score & IRS classification \\
\hline no positive cell $(0)$ & no reaction $(0)$ & negative $(0-1)$ & negative $(0)$ \\
\hline$<10 \%$ positive cells $(1)$ & mild reaction (1) & mild $(2-3)$ & positive, weak exp. (1) \\
\hline $10-50 \%$ positive cells (2) & moderate reaction (2) & moderate $(4-8)$ & positive, intermediate exp. (2) \\
\hline $51-80$ positive cells ( 3 ) & strong reaction $(3)$ & strong (9-12) & positive, strong exp. (3) \\
\hline$>80 \%$ positive cells $(4)$ & & & \\
\hline
\end{tabular}

*IRS score - Percentage of positive cells $\times$ Staining intensity, exp - expression

TNM classification (American Joint Committee on Cancer, 2010) (5). Immunohistochemical analysis of ADAMTS1, ADAMTS8, and ADAMTS18 was performed by using paraffin-embedded samples of the cases. Patients' noncancerous gastric tissues and nonmetastatic lymph node tissues were used as control. The immunohistochemistry was evaluated by at least two trained pathologists.

Written informed consent was obtained from all patients, and the study protocol was approved by the Medical Ethics Committee of Turgut Ozal University, Faculty of Medicine, Turkey (Permit Number, date: 99950669/359, 28/11/2014).

\section{Immunohistochemistry}

All experimental steps were performed in accordance with the protocols recommended for the anti-human ADAMTS1, 8, and 18 polyclonal antibodies (Abcam). After being deparaffinized at

Tab. 2. Patient demographics and tumor characterictics $(n=25)$.

\begin{tabular}{|c|c|}
\hline Parameters & $\mathrm{n}(\%)$ \\
\hline Age (y) & $63.6 \pm 6.9(42-73 \mathrm{y})$ \\
\hline \multicolumn{2}{|l|}{ Gender } \\
\hline Male & $15(60 \%)$ \\
\hline Female & $10(40 \%)$ \\
\hline \multicolumn{2}{|l|}{ Localization of tumor } \\
\hline Proximal (cardia/fundus) & $4(16 \%)$ \\
\hline corpus & $11(44 \%)$ \\
\hline Distal (antrum/pylorus) & $10(40 \%)$ \\
\hline \multicolumn{2}{|l|}{ Type of gastric resection } \\
\hline subtotal & $10(40 \%)$ \\
\hline total & $15(60 \%)$ \\
\hline \multicolumn{2}{|l|}{ Type of lymph node dissection } \\
\hline D1 dissection & $7(28 \%)$ \\
\hline D2 dissection & $18(72 \%)$ \\
\hline \multicolumn{2}{|l|}{ Lauren classification } \\
\hline Intestinal & $16(64 \%)$ \\
\hline Diffuse & $6(24 \%)$ \\
\hline Undetermined & $3(12 \%)$ \\
\hline Tumor size (mm, mean) & $26.2 \pm 5.8(19-34 \mathrm{~mm})$ \\
\hline Total number of lymph nodes & $37.6 \pm 18.4(19-72)$ \\
\hline Number of metastatic lymph nodes & $8 \pm 6.4(0-19)$ \\
\hline Tumor stage & Stage $2(5,20 \%)$, Stage $3(20,80 \%)$ \\
\hline \multicolumn{2}{|l|}{ Tumor differentiation grade } \\
\hline Well-differentiated & $19(76 \%)$ \\
\hline Moderately-differentiated & $6(24 \%)$ \\
\hline Vascular invasion positivity & $15(60 \%)$ \\
\hline Lymphatic invasion positivity & $10(40 \%)$ \\
\hline Perineural invasion positivity & $10(40 \%)$ \\
\hline
\end{tabular}

$65{ }^{\circ} \mathrm{C}$ in heat chamber and rehydrated, sections were subjected epitope retrieval in 10X EDTA buffer $\left(\mathrm{pH} \mathrm{8.0)}\right.$ ) at $110{ }^{\circ} \mathrm{C}$ for 30 min. Subsequently, the sections were exposed to $3 \% \mathrm{H}_{2} \mathrm{O}_{2}$ for $20 \mathrm{~min}$ to bleach endogenous peroxidases, and were rinsed three times with phosphate-buffered saline (PBS) for $10 \mathrm{~min}$. Sections were incubated with a rabbit anti-human ADAMTSs (all 1:250 in BSA) for $1 \mathrm{~h}$ at $37^{\circ} \mathrm{C}$, washed three times in PBS and incubated in a biotinylated goat secondary anti-mouse polyclonal antibody for $15 \mathrm{~min}$ at $37^{\circ} \mathrm{C}$. After being washed in PBS, the tissues were visualized with 3,3'-diaminobenzidine tetrahydrochloride (DAB chromogen, Abcam) and counterstained with hematoxylin. Finally, the sections were dehydrated in graded ethanol, immersed in xylene and coverslipped. All images were acquired using a 40X objective and a microscope (Leica).

\section{Evaluation of immunostaining}

Immunoreactivity of ADAMTS1, 8, and 18 in all samples was evaluated using a well-established immunoreactivity scoring system (IRS) which takes into account both the percentage of positive cells and staining intensity (6). All tissues were scored between 0 (no staining) and 12 (maximum staining) according to IRS (Tab. 1). All ADAMTS expressions were scored by two pathologists blinded to clinical details for each case. In addition to intensity of staining, intra/extracellular distribution of staining (cytoplasm, nucleus, and surrounding stroma) was also evaluated. All statistical analyses between immunostaining status of ADAMTSs and the clinicopathological parameters were performed by using the mean IRSs of ADAMTSs in cancerous and healty tissues.

\section{Statistical analysis}

All data were statistically analyzed by using the statistical package for social sciences (SPSS 21.0 software, IL-ChicagoUSA). Results of descriptive analysis were expressed as the mean $\pm \mathrm{SD}$ and/or number (percentages) for variables. The expression profiles (IRS scores) of ADAMTS1, 8, and 18 between cancerous and non-cancerous tissues, and the relationship between ADAMTS1, 8, and 18 immunostaining status and the histopathological characteristics were assesed by using Pearson Chi-Square, Fisher's Exact Test and Spearman's correlation test. Significance level was accepted as $\mathrm{p}<0.05$.

\section{Results}

\section{Perioperative data}

A total of 25 patients (mean age 63.6 years) who underwent curative resection for gastric adenocarcinoma were included in 
Tab. 3. Mean IRS scores of ADAMTS1, 8, and 18 in cancerous and non-cancerous tissues.

\begin{tabular}{|c|c|c|c|c|c|c|}
\hline & ADAMTS & 1 & ADAMTS & 8 & ADAMTS & 18 \\
\hline & IRS & $\mathrm{p}$ & IRS & $\mathrm{p}$ & IRS & $\mathrm{p}$ \\
\hline NCGT & $\begin{array}{c}1.32 \pm 1.4 \\
(0-6)\end{array}$ & $<0.001$ & $\begin{array}{c}0.56 \pm 0.8 \\
(0-3)\end{array}$ & $<0.001$ & $\begin{array}{l}1 \pm 0.9 \\
(0-3)\end{array}$ & $<0.001$ \\
\hline$\overline{\mathrm{CGT}}$ & $\begin{array}{c}5.68 \pm 2.8 \\
(2-12)\end{array}$ & & $\begin{array}{c}3.1 \pm 1.5 \\
(1-6)\end{array}$ & & $\begin{array}{l}6.5 \pm 3.4 \\
(2-12)\end{array}$ & \\
\hline NMLN & $\begin{array}{c}0.84 \pm 0.8 \\
(0-3)\end{array}$ & $<0.001$ & $\begin{array}{c}1.6 \pm 1.5 \\
(0-6)\end{array}$ & $<0.001$ & $\begin{array}{c}0.92 \pm 1.0 \\
(0-3)\end{array}$ & $<0.001$ \\
\hline$\overline{\mathrm{MLN}}$ & $\begin{array}{c}5.4 \pm 3.3 \\
(0-12)\end{array}$ & & $\begin{array}{c}2.3 \pm 1.7 \\
(0-8)\end{array}$ & & $\begin{array}{c}6.28 \pm 2.9 \\
(2-12)\end{array}$ & \\
\hline
\end{tabular}

IRS scores are presented as mean \pm SD (minimum-maximum), NCGT - Non-cancerous gastric tissue, CGT v Cancerous gastric tissue, NMLN - Non-metastatic lymph node, MLN - Metastatic lymph node

the study. Demographic data and tumor characteristics are presented in Table 2. Total gastrectomy was the most common operation type, and comnplications related to surgery were seen in three patients (two with enterocutaneous fistula and one with intraabdominal hemorrhage). All complications were successufully treated conservatively, and no re-operation was needed. There was no death within the postoperative period of one month. All patients were followed-up regularly. The median overall survival was 32 months (8-63), and only five (20\%) patients survived over four years after surgery.

Expression status of ADAMTS1,8, and 18 in cancerous and noncancerous tissues

It was clearly revealed that GC expressed ADAMTS1, 8, and 18 in all samples, with different IRS scores. The mean IRS scores of three ADAMTS proteases in cancerous and non-cancerous tissues are presented in Table 3.

Positive immunostaining profiles of ADAMTS1, 8, and 18 were observed in the cytoplasm of the cancer cells and surrounding stromal tissues, and the IRS scores of all ADAMTSs were significantly higher in the cancerous gastric tissue in comparison to

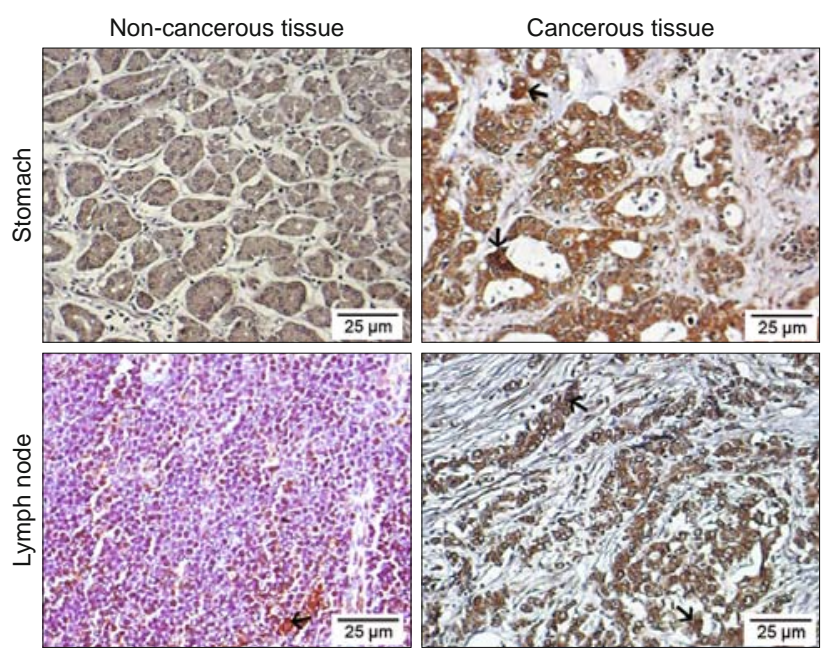

Fig. 1. Immunohistochemical illustrations of gastric and lymph node tissues of ADAMTS1. Arrows show positive staining areas, streptavidine-peroxidase. (Scale bar $=25 \mu \mathrm{m})$. non-cancerous gastric tissue $(\mathrm{p}<0.001)$. In addition, IRS scores of the three ADAMTS proteases were higher in the metastatic lymph nodes compared with healthy lymph nodes $(\mathrm{p}<0.001)$ (Figs. 1-3).

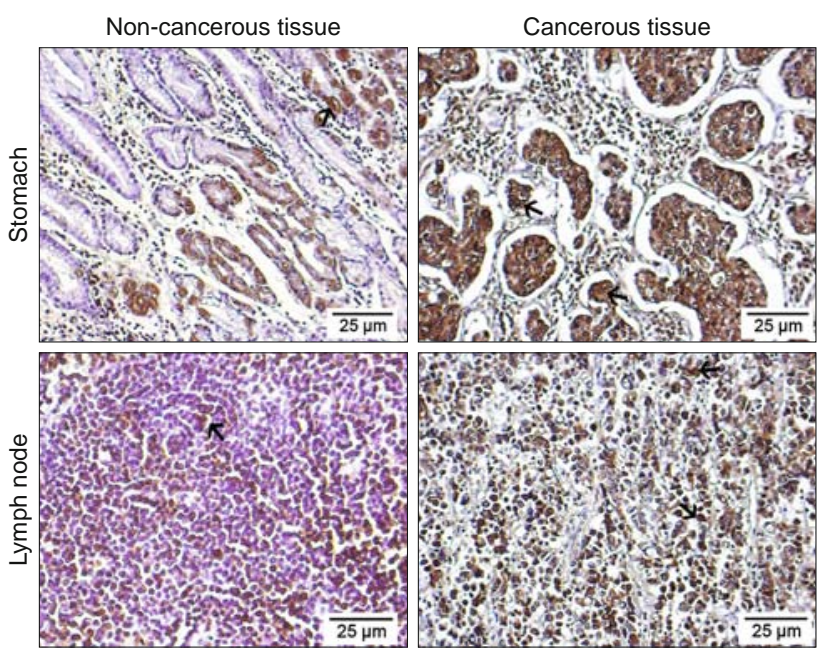

Fig. 2. Immunohistochemical illustrations of gastric and lymph node tissues of ADAMTS8. Arrows show positive staining areas, streptavidine-peroxidase. (Scale bar $=25 \mu \mathrm{m})$.

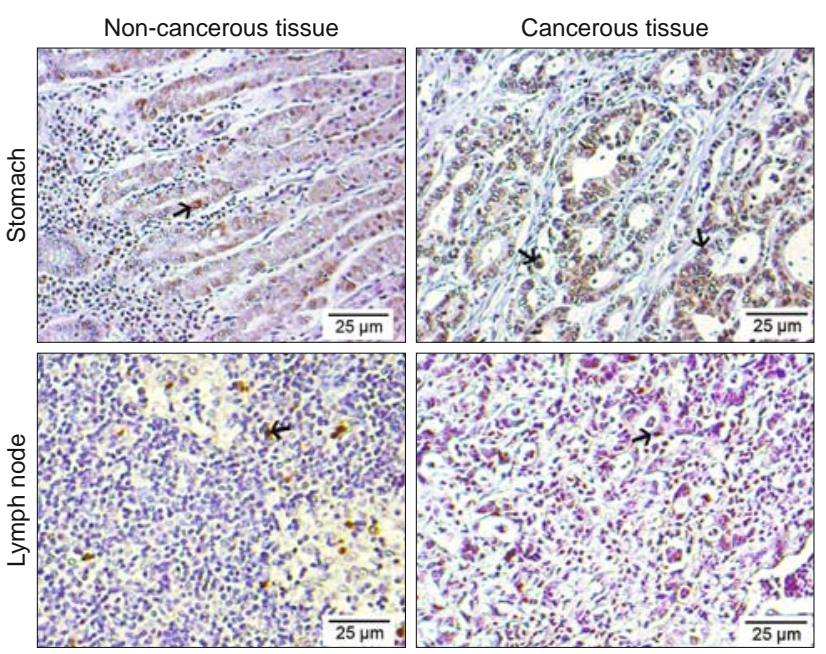

Fig. 3. Immunohistochemical illustrations of gastric and lymph node tissues of ADAMTS18. Arrows show positive staining areas, streptavidine-peroxidase. $($ Scale bar $=\mathbf{2 5} \mu \mathrm{m})$. 
Correlation of ADAMTS1,8, and 18 expressions and tumor characteristics

The expression status of ADAMTS1 in cancerous gastric tissue showed positive correlation with grade (rho: 0.661, $\mathrm{p}<0.001$ ) and stage (rho: $0.649, \mathrm{p}<0.001$ ). A positive correlation between ADAMTS8 expression level in cancerous gastric tissue and two parameters was also found: tumor size (rho: 0.658, $\mathrm{p}<0.001$ ) and stage (rho: $0.532, \mathrm{p}<0.001$ ). On the other hand, the expression of ADAMTS18 in cancerous gastric tissue was found to be only correlated with stage of tumor (rho: $0.529, \mathrm{p}<0.001$ ). There were no correlations between the three ADAMTSs and the other clinicopathological parameters $(\mathrm{p}>0.05)$.

\section{Discussion}

It is well known that carcinogenesis is a multi-step complex process involving various environmental and genetic factors (7). In addition, many abnormal events in the tumor microenvironment such as loss of cell cycle control, changes in apoptotic and angiogenic functions, and destruction of ECM contribute to cancer growth and metastasis. Therefore, most researchs have focused on understanding the molecular biology and behavior of cancer in recent years. In this context, ADAMTS family, as a relatively new group of ECM metalloproteinases, have become one of the promising foci in the recent studies on cancer. To date, many clinical studies regarding the relationship between ADAMTS proteases and various types of cancer have been reported (7-10). However, there are limited data on the role of ADAMTSs in gastric cancer. In this study, we demonstrated high expression status of ADAMTS1, 8, and 18 in gastric adenocarcinoma immunohistochemically.

In the literature, ADAMTS1, the first member of ADAMTS family discovered by Kuno in a cell model of colon cancer cachexia, is the best described protein among all ADAMTSs (11). While aggrecanase activity specific to some ECM substrats including aggrecan and versican is the leading property of $\mathrm{AD}$ AMTS1, it has also a potent anti-angiogenic property which was first identified by Vazquez et al (12). Its antiangiogenic effect occurs through several mechanisms such as binding to FGF-2, sequestration of VEGF165 and some bioactive antiangiogenic substrates that are liberated as a result of TS- 1 and TS-2 proteolysis. The anti-tumoral effects of ADAMTS1 through antiangiogenic activity were shown in suppressing vascularization of liver metastases (13). ADAMTS1 has been found to play significiant roles in many benign and malignant diseases. In various malignancies, ADAMTS1 has both protumoral and anti-tumoral effects (7). High ADAMTS1 expression level was shown in cervical cancer and melanoma (12). Overexpression of ADAMTS1 was also found in pancreatic cancer, suggesting to be associated with local invasion and lymphatic metastases (14). In a study from China, ADAMTS1 gene expression was found over-expressed in primary gastric cancer at mRNA level. The authors also showed over-expression of ADAMTS1 in precancerous lesions including intestinal metaplasia and dysplasia indicated that this gene might be a potential biomakers for early detection of GC (15). In another study aimed to investigate the possible correlation of ADAMTS1 with angiogenesis in GC, it was shown that ADAMTS1 had angioinhibitory effects in primary gastric cancer due to its low expression and negative correlation with VEGF and microvessel density (16). According to our results, ADAMTS1 expression was significiantly higher in cancerous gastric tissue and metastatic lymph nodes compared with healthy tissues, suggesting this protease may be involved in tumor growth and lymphatic metastasis. It should be stated here that ADAMTS1 has both pro- and antitumoral effects, but these functions and underlying mechanisms remain largely unknown due to the limited knowledge about this protein.

The second protease investigated in the present study was ADAMTS8 which is characterized by anti-angiogenic effects similar to ADAMTS1. This protease was previously shown to be downregulated in various types of cancer such as breast, brain, and non-small cell lung carcinomas (17-19). Porter at al also found in their study that high expression levels of ADAMTS8 together with low expression levels of ADAMTS15 were associated with poor prognosis in breast cancer (17). To the best of our knowledge, there are only two clinical studies on the potential role of ADAMTS8 in GC $(20,21)$. Both studies focused on the angioinhibitory effects of ADAMTS8, and mainly examined the methylation status of this protease. Differently from these works, we investigated the expression profile of ADAMTS8 and its relationship with clinicopathological factors by using immunohistochemical method. In one of these studies, it was found that ADAMTS8 was downregulated or silenced by promoter methylation in gastric carcinoma cell lines. The authors also demonstrated that this metalloprotease acts as a functional tumor suppressor through antagonizing EGFR-MEK-ERK signaling, and inhibits tumor cell motility (20). The other study, reported by Chen et al, showed that ADAMTS8 hypermethylation is associated with decreased expression in GC and may play an important role in the invasion and metastasis of GC (21). According to the findings obtained from our study, positive immunostaining of ADAMTS8 in gastric cancerous tissue and lymph node was higher than in adjacent normal gastric and lymphatic tissues. In addition, we found that ADAMTS8 expression in cancerous gastric tissue was correlated with tumor size and stage. These results suggest that ADAMTS8 may have important roles in carcinogenesis and lymphatic spread of tumor cells. However, it is stil an enigma how the balance between pro-tumoral activities and angioinhibitory effects is regulated in the behaviour of cancer cells.

Finally, we analyzed the immunostaining status of ADAMTS18 in the present study. This protein is normally expressed by endothelial cell in various human tissues including brain, esophagus, stomach, colon and pancreas. ADAMTS18 has two important properties: being an aggrecanase and maintenance of homeostasis via activation of platelet aggregation. Its aggrecanese property is rather low and manifests itself at high concentrations of ADAMTS18 (22). The second function is considered to play role in the inbition of metastasis through distruption of tumor emboli $(4,23)$. Reduced and/or totally silenced expression of ADAMTS18 in multiple cancer cell lines and mutation 
and/or deletion of this gene in various cancers such as breast and colorectal cancers were demonstrated in previous reports $(24$, 26). To our knowledge, there is only one study investigating the assocation of ADAMTS18 and GC in the current literature (27). In that study, high-resolution melting analysis was used to detect the methylation levels of ADAMTS18 gene in three types of cancer; colorectal cancer, pancreatic cancer, and GC. The frequency of ADAMTS18 methylation in all three types of cancer was found to be significantly higher than that in normal tissues. In addition, the authors found expression levels of ADAMTS18 were inversely correlated with methylation levels, and there was no association between ADAMTS18 methylation status and TNM staging of cancer. We used immunochemistry first time in the literature to investigate the expression status of ADAMTS18 in GC. According to our results, ADAMTS18 expression was higher in tumoral tissue and metastatic lymph nodes compared with the non-cancerous tissues. In addition, ADAMTS18 expression was found to be correlated with stage of tumor, similar to ADAMTS1 and ADAMTS8. In our opinion, these results may indicate the potential role of ADAMTS18 in carcinogenesis, local invasion and lymphatic spread of gastric cancer cells.

This study has several limitations. First of all, it was conducted in a single center, which may limit the generalizability of the results. A relatively small sample size is another limitation of this work, which make it difficult to interpret subgroup findings. Finally, using a single experimental method may be considered as a limitation. However, filling a gap in this field can make this study valuable.

In conclusion, this study clearly showed that ADAMTS1, 8, and 18 are highly expressed in GC and its nodal metastases, suggesting important roles of these proteases in carcinogenesis and lymphatic metastasis. The positive correlation between these three ADAMTSs and stage of tumor support this view. The findings from the present study may indicate that these proteases may be promising candidates for novel and alternative treatments in GC.

\section{Learning points}

1. ADAMTS proteases are involved in various important biological processes such as extracellular matrix remodeling and the regulation of angiogenesis associated with carcinogenesis and metastasis.

2. ADAMTS1, 8, and 18 are classified as aggrecanases. ADAMTS1 and 8 have anti-angiogenic property while ADAMTS18 is known as a tumor suppressor gene.

3. ADAMTS1, 8, and 18 are highly expressed in gastric cancer and its nodal metastases, suggesting important roles of these proteases in carcinogenesis and lymphatic metastasis.

4. According to the results obtained from the study, these proteases may be promising candidates for novel and alternative treatments in gastric cancer.

\section{References}

1. Jemal A, Bray F, Center MM, Ferlay J, Ward E, Forman D. Global cancer statistics. CA. Cancer J Clin 2014; 61 (2): 69-90.

2. Howlader N, Noone AM, Krapcho M et al. National Cancer Institute. SEER Cancer Statistics Review, 1975-2010 [Internet] Bethesda (MD): National Cancer Institute; [cited 2013 Apr]. Available from: http://seer. cancer.gov/archive/csr/1975_2010/.

3. Niccolai E, Taddei A, Prisco D, Amedei A. Gastric cancer and the epoch of immunotherapy approaches. World J Gastroenterol 2015; 21 (19): 5778-5793.

4. Wagstaff L, Kelwick R, Decock J, Edwards DR. The roles of ADAMTS metalloproteinases in tumorigenesis and metastasis. Front Biosci 2011; 16: 1861-1872.

5. Edge SBBD, Compton CC, Fritz AG, Greene FL, Trotti A. AJCC Cancer Staging Manual. Springer; New York, NY: 2010.

6. Remmele W, Stegner HE. Recommendation for uniform definition of an immunoreactive score (IRS) for immunohistochemical estrogen receptor detection (ER-ICA) in breast cancer tissue. Pathologe 1987; 8 (3): 138-140.

7. Cal S, López-Otín C. ADAMTS proteases and cancer. Matrix Biol 2015; 44-46C: 77-85.

8. Kumar S, Rao N, Ge R. Emerging Roles of ADAMTSs in Angiogenesis and Cancer. Cancers (Basel) 2012; 4 (4): 1252-1299.

9. Uysal S, Unal ZN, Erdogan S et al. Augmentation of ADAMTS9 gene expression by IL- $1 \beta$ is reversed by NFKB and MAPK inhibitors, but not PI3 kinase inhibitors. Cell Biochem Funct 2013; 31 (7): 539-544.

10. Filou S, Korpetinou A, Kyriakopoulou D et al. ADAMTS Expression in Colorectal Cancer. PLoS One 2015; 10 (3): e0121209.

11. Kuno K, Kanada N, Nakashima E, Fujiki F, Ichimura F, Matsushima K. Molecular cloning of a gene encoding a new type of metalloproteinase-disintegrin family protein with thrombospondin motifs as an inflammation associated gene. J Biol Chem 1997; 272 (1): 556-618.

12. Vazquez F, Hastings G, Ortega MA et al. METH-1, a human ortholog of ADAMTS-1, and METH-2 are members of a new family of proteins with angio-inhibitory activity. J Biol Chem 1999; 274 (33): 23349-23357.

13. Lee YJ, Koch M, Karl D et al. Variable inhibition of thrombospondin 1 against liver and lung metastases through differential activation of metalloproteinase ADAMTS1. Cancer Res 2010; 70 (3): 948-956.

14. Masui T, Hosotani R, Tsuji S et al. Expression of METH-1 and METH-2 in pancreatic cancer. Clin Cancer Res 2001; 7 (11): 3437-3443.

15. Guo RF, Zang SZ, Fang JY et al. Identification of biomarkers for early detection in gastric cancer and its clinical biological significance. Beijing Da Xue Xue Bao 2009; 41 (3): 353-360.

16. Chen J, Zhi Y, Chang X, Zhang S, Dai D. Expression of ADAMTS1 and its correlation with angiogenesis in primary gastric cancer and lymph node metastasis. Dig Dis Sci 2013; 58 (2): 405-413.

17. Porter S, Scott SD, Sassoon EM et al. Dysregulated expression of adamalysin-thrombospondin genes in human breast carcinoma. Clin Cancer Res 2004; 10 (7): 2429-2440.

18. Rocks N, Paulissen G, Quesada Calvo F et al. Br J Cancer 2006; 94: 724-730.

19. Dunn JR, Reed JE, du Plessis DG et al. Expression of ADAMTS-8, a secreted protease with antiangiogenic properties, is downregulated in brain tumors. Br J Cancer 2006; 94 (5): 1186-1193. 


\section{1-76}

20. Choi GC, Li J, Wang Y et al. The metalloprotease ADAMTS8 displays antitumor properties through antagonizing EGFR-MEK-ERK signaling and is silenced in carcinomas by CpG methylation. Mol Cancer Res 2014; 12 (2): 228-238.

21. Chen J, Zhang J, Li X et al. Downregulation of ADAMTS8 by DNA Hypermethylation in Gastric Cancer and Its Clinical Significance. Biomed Res Int 2016; 2016: 5083841.

22. Zeng W, Corcoran C, Collins-Racie LA et al. Glycosaminoglycanbinding properties and aggrecanase activities of truncated ADAMTSs: comparative analyses with ADAMTS-5, $-9,-16$ and -18 . Biochim Biophys Acta 2006; 1760 (3): 517-524.

23. Li Z, Nardi MA, Li YS et al. C-terminal ADAMTS-18 fragment induces oxidative platelet fragmentation, dissolves platelet aggregates, and protects against carotid artery occlusion and cerebral stroke. Blood 2009; 113 (24): 6051-6060.
24. Jin H, Wang X, Ying $\mathbf{J}$ et al. Epigenetic identification of ADAMTS18 as a novel 16q23.1 tumor suppressor frequently silenced in esophageal, nasopharyngeal and multiple other carcinomas. Oncogene 2007; 26 (53): 7490-7498.

25. Sjoblom T, Jones S, Wood LD et al. The consensus coding sequences of human breast and colorectal cancers. Science 2006; 314 (5797): 268-274.

26. Nordgard SH, Johansen FE, Alnaes GI et al. Genome-wide analysis identifies $16 \mathrm{q}$ deletion associated with survival, molecular subtypes, mRNA expression, and germline haplotypes in breast cancer patients. Genes Chromosomes Cancer 2008; 47 (8): 680-696.

27. Li Z, Zhang W, Shao Y et al. High-resolution melting analysis of ADAMTS18 methylation levels in gastric, colorectal and pancreatic cancers. Med Oncol 2010; 27 (3): 998-1004. 